\title{
Ileal intussusception due to primary intestinal melanoma with generalized lymph node metastases- a rare cause of small bowel obstruction
}

\section{Keerthi Andi Rajendharan, Gurushankari Balakrishnan, Sudharsanan Sundaramurthi*, Balamourougan Krishnaraj, Sarath Chandra Sistla}

Department of Surgery, Jawaharlal Institute of Postgraduate Medical Education and Research, Puducherry, India

Received: 18 January 2021

Revised: 19 January 2021

Accepted: 20 January 2021

\section{*Correspondence:}

Dr. Sudharsanan Sundaramurthi,

E-mail: sudharsanans4@gmail.com

Copyright: () the author(s), publisher and licensee Medip Academy. This is an open-access article distributed under the terms of the Creative Commons Attribution Non-Commercial License, which permits unrestricted non-commercial use, distribution, and reproduction in any medium, provided the original work is properly cited.

\begin{abstract}
Primary melanomas of small intestine are rare and most of them are metastases from cutaneous melanoma. The features distinguishing primary from metastatic intestinal melanoma are still under debate. Primary intestinal melanoma (PIM) is associated with a worse prognosis and a more aggressive behaviour due to its rapid growth. Hence, we report a case of primary ileal melanoma presenting as intussusception with generalized lymph node metastasis. A 69-year-old lady presented with recurrent abdominal pain, vomiting, distension and low grade fever. On examination, a $4 * 4 \mathrm{~cm}$ right inguinal lymph node was noted with no abnormalities on abdominal and per rectal examination. Computed tomography (CT) revealed ileoileal intussusception which was confirmed on laparotomy and diagnosed as primary ileal melanoma on histopathological examination (HPE). Whole-body Positron Emission Tomography (PET) CT revealed metastasis to lymph nodes all over the body. Intestinal melanomas are rare and mostly occur secondary to primary cutaneous melanoma. The clinical picture of intestinal melanoma is varied, ranging from chronic vague symptoms to surgical emergency such obstruction and bleeding. Treatment of both primary as well as metastatic intestinal melanoma is surgical excision. Intestinal melanoma, in general, carries a bad prognosis than cutaneous melanoma. Owing to the rarity and vague presentation, early diagnosis of intestinal melanoma requires a high index of suspicion, multimodal imaging and timely surgical intervention with adequate resection margins.
\end{abstract}

Keywords: Gastrointestinal malignancy, Inguinal lymph node, Cutaneous melanoma, Mesenteric nodes, Palliative chemotherapy

\section{INTRODUCTION}

Cutaneous malignant melanoma is one of the most common malignancies in the world. However, primary non-cutaneous melanoma is unusual and most commonly found to be of ocular, mucosal and leptomeningeal sites. ${ }^{1}$ Malignant melanoma of gastrointestinal tract (GIT) accounts to 1 to $3 \%$ of all GI malignancies, most of which represent secondaries from extra-GI primaries. ${ }^{2}$ Though primary intestinal melanomas (PIM) have been previously described in the literature, occurrence of these lesions in the GIT apart from oesophagus and rectum where normally melanocytes exists is still a subject of debate. Also distinguishing, PIM from metastatic intestinal melanoma (MIM) is challenging and both the entities carry a worse prognosis when compared to their primary cutaneous counterpart. ${ }^{3}$ Herein, we report a case of primary ileal malignant melanoma presenting as intussusception along with generalized lymphadenopathy. 


\section{CASE REPORT}

A 69-year-old lady presented with complaints of recurrent diffuse abdominal pain andbilious vomiting of one-month duration. It was associated with low-grade intermittent fever and abdominal distension. Her general physical examination revealed pallor and a single $4 * 4 \mathrm{~cm}$ hard right superficial inguinal lymphadenopathy. Her abdomen was distended with diffuse tenderness and guarding. Rectal examination was unremarkable.

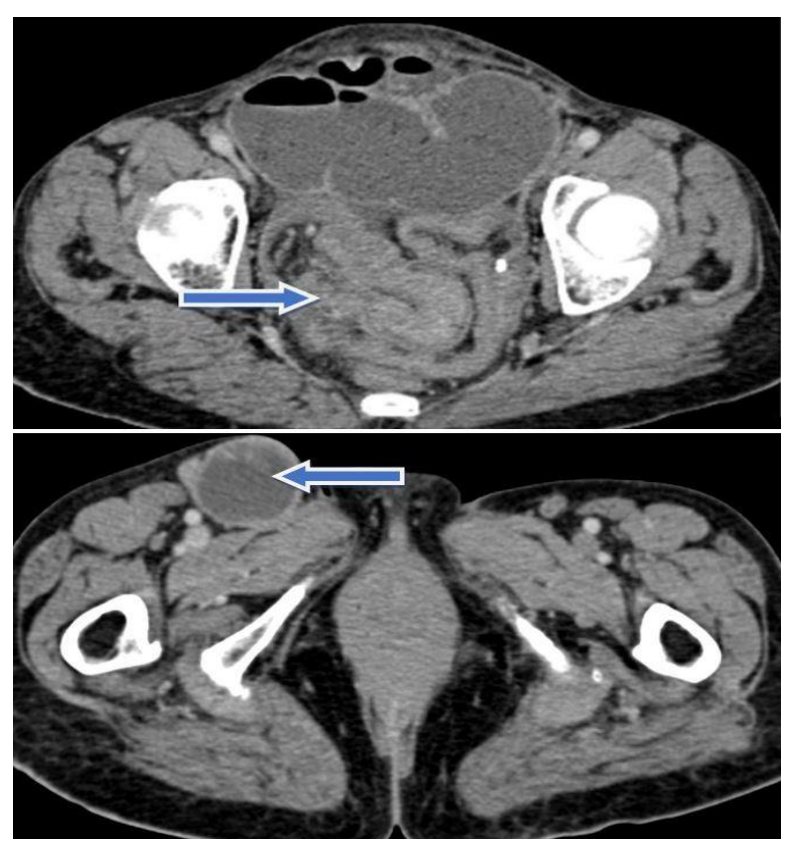

Figure 1: Contrast enhanced computed tomography(A) axial section of abdomen showing ileo-ileal intussusception (arrow), (B) metastatic right inguinal lymph node.
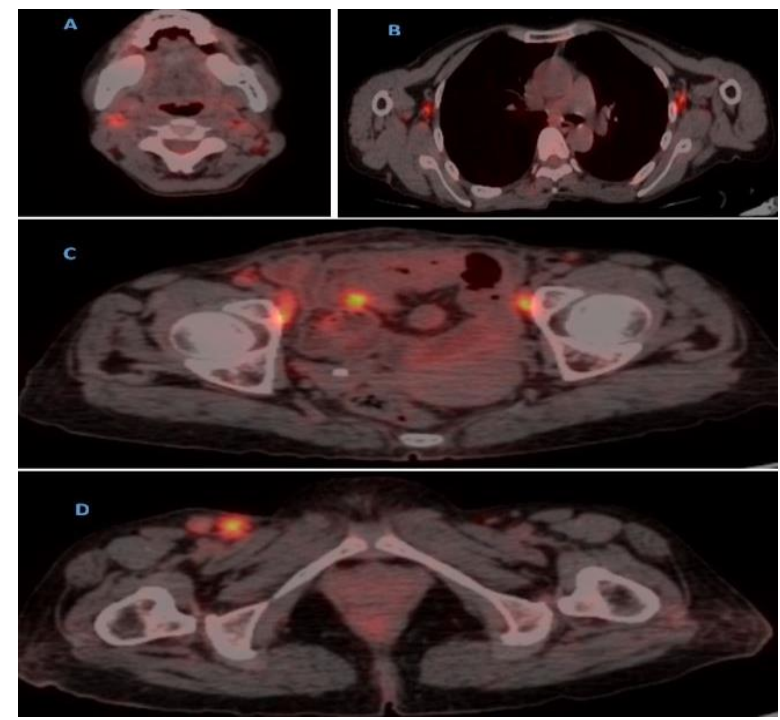

Figure 1: ${ }^{18}$ FDG PET scan shows metabolically active bilateral cervical (A), bilateral axillary (B), bilateral external iliac (C), and bilateral inguinal lymph nodes (D) suggestive of metastases.
Her abdominal radiograph revealed dilated small bowel loops with multiple air-fluid levels and ultra-sonogram (USG) abdomen revealed evidence of bowel within bowel appearance in the right iliac fossa, suggestive of ileo-ileal intussusception. A contrast enhanced computer tomography (CECT) revealed ileo-ileal intussusception, ileal mass lesion as the lead point with dilated proximal bowel loops, suggestive of small bowel obstruction. Associated mesenteric lymph node enlargement and right inguinal lymphadenopathy were noted. Her laboratory work-up revealed anaemia with normal renal and liver functions.

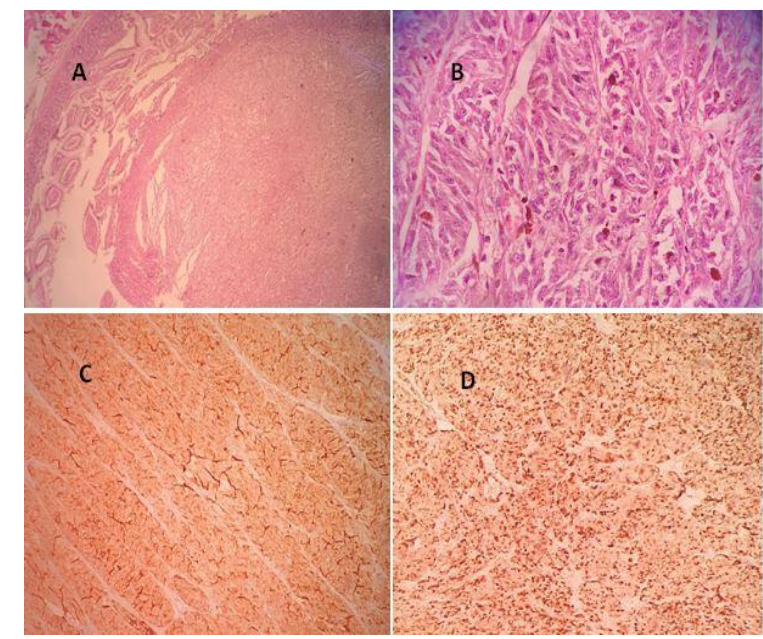

Figure 3: Histopathological examination showing (A) nests of tumour cells in the ileum; 10X, (B) oval to polygonal tumour cells with abundant cytoplasm and prominent nucleoli with presence of melanin pigment;

40X, (C, D) positive immunohistochemistry for melanin A and HMB45.

She underwent emergency laparotomy; intra-operatively an ileo-ileal intussusception was noted $80 \mathrm{~cm}$ proximal to the ileocaecal junction, which revealed healthy bowel and a mass lesion arising from the wall of the distal ileum of size $5 * 4 * 2 \mathrm{~cm}$ as a lead point with healthy intussusceptum on milking it out. Resection of the mass with $10 \mathrm{~cm}$ proximal and distal margins and ileo-ileal anastomosis was performed. The inguinal lymph node mass was also excised.

Histopathology examination (HPE) revealed primary malignant mucosal melanoma of ileum, resected margins free of tumour, with pericolic nodal metastases and metastatic malignant melanoma of the excised inguinal lymph node. On immunohistochemistry (IHC), the tumour cells were positive for HMB45, Melan-A, S-100 and negative for SOX-10, CD-117 and CK.

A fluoro-deoxyglucose-positron emission tomographycomputer tomography (FDG-PET-CT) was done, which revealed metabolically active regions in bilateral cervical, left supraclavicular, bilateral axillary, bilateral external iliac, bilateral inguinal lymph nodes and spleen, 
suggestive of metastases. She was planned for palliative chemotherapy in view of metastatic intestinal melanoma.

\section{DISCUSSION}

Malignant melanoma is defined as a neoplastic transformation of melanocytes which are typically found in the skin, the eye, and the nose. Melanoma occurring in the gastrointestinal tract is unusual and accounts to 1 to $3 \%$ of the entire spectrum of GI malignancy. ${ }^{4}$

PIM can occur anywhere within the GI mucosa, but is most commonly detected in the anorectal and oropharyngealregions $(32.8 \%)$, whereas esophagus $(5.9 \%)$, small intestine $(2.3 \%)$, gallbladder $(1.4 \%)$, and large intestine $(0.9 \%)$ are rare sites of origin. ${ }^{5}$ Small bowel is the most common site for metastasis from undetected or spontaneously regressed cutaneous melanomas, accounting to $2 \%$ of all the small bowel malignancies. ${ }^{6}$

PIM is an extremely rare entity for which cause is not known yet. According to Mishima et al the disease has been postulated to arise from neuroblast cells associated with autonomic innervation of GIT. ${ }^{7}$ Amar et al. postulated that melanoblastic cells of the neural crest, which migrate to the distal ileum through the omphalomesenteric canal result in origin of PIM. ${ }^{8}$ The amine-precursor uptake and decarboxylation cell concept states that PIM arise due to neoplastic transformation in non-cutaneous sites. However, Leschet al metastasis to the small intestine has been established due to the aberrant cell surface expression of chemokine receptor type. ${ }^{9}$ This receptor is selectively expressed in the small intestine and thymus and its regulation is likely to be a crucial step in the metastatic process. ${ }^{9}$

A diagnosis of PIM is made when HPE shows a precursor lesion or melanosis of intestinal mucosa. Men are affected more than women and most commonly noted as solitary lesion in the ileum. PIM is more aggressive and is associated with a worse prognosis than cutaneous melanoma mainly due to late diagnosis and rapid growth because of the rich lymphovascular supply of the intestinal mucosa. ${ }^{3}$

Metastatic intestinal melanomas (MIM) are more common than PIM. In contrast to PIM, MIM forms multiple polypoidal masses that equally affect jejunum and ileum. These lesions can be pigmented or amelanotic and more commonly ulcerate. ${ }^{3}$ Bender et al described four different types of MIM of small bowel: polypoid, infiltrating, cavitary and exoenteric. ${ }^{10}$ MIM is often undetectable in early stages and is diagnosed only when complications occur or remain asymptomatic until diagnosed during autopsy. Though metastasis to the small bowel manifests 3-6 years after removal of the cutaneous lesions, sometimes they are present at initial diagnosis or within 6 months after diagnosis of cutaneous lesions. ${ }^{11}$ The time period between excision of cutaneous melanoma and the diagnosis of MIM ranges between 6 to 90 months. $^{12}$

The clinical presentation of intestinal melanoma is identical to that of other gastrointestinal tumours. ${ }^{3}$ The most common symptoms are abdominal pain, constipation, melena, anaemia, fatigue, weight loss, and the presence of a palpable abdominal mass. They can also rarely present as single or multiple polyps causing intestinal intussusception and obstruction. Significant intraabdominal lymphadenopathy indicates spread to the mesenteric lymph nodes. ${ }^{13}$ Clinically, looking for primary cutaneous lesions with a head-to-toe examination, generalized lymphadenopathy and systemic examination is prudent. Our patient had a similar symptom complex comprising of vague and recurrent abdominal pain, vomiting and on and off distension of abdomen for one month and presented to us in emergency department with intussusception causing intestinal obstruction.

Typically, abdominal ultrasonography is the first investigation for patients presenting vague symptoms as it is non-invasive and widely available with the possibility for guided biopsy from the suspicious lesion or lymph nodes. ${ }^{14}$ Other imaging modalities include barium studies, multidetector computed tomography (MDCT) and CT enteroclysis. Endoscopic evaluation of small bowel using double-balloon enteroscopy and capsule endoscopy has also been described. ${ }^{3}$ PET imaging has higher sensitivity and specificity than CECT for detection of GI metastases in patients with melanoma. ${ }^{15}$ In our patient while, preoperative CECT abdomen and pelvis showed only intussusception with dilated small bowel loops and enlarged right inguinal nodes, PET-CT done postoperatively revealed extensive metastasis to lymph nodes in cervical, axillary and inguinal regions with deposits in the spleen. Hence, patients with a history of cutaneous melanoma and obscure gastrointestinal bleeding, anaemia, and other non-specific abdominal symptoms, a combination of imaging techniques is recommended for early diagnosis of small-bowel metastases.

Surgery is the treatment of choice for both primary as well as metastatic intestinal melanoma. Wide surgical excision with adequate proximal and distal free margins and wedge resection of mesentery down to the root for adequate lymph node harvest is preferred. Nevertheless, surgical resection of metastases to small bowel is proposed only among patients in whom complete removal of the disease is feasible. ${ }^{16}$ Hence, in our patient $10 \mathrm{~cm}$ proximal and distal margin were given with wedge resection of mesentery till the root and the suspicious right inguinal lymph node was excised and sent for HPE which was reported as PIM with free margins, one out of the 3 lymph nodes in the mesentery were positive and the right inguinal lymph node was reported to harbor metastatic deposits. 
The median survival following complete tumour resection in MIM is 15 months while complete resection with negative margins in PIM substantially improves the prognosis with a 5-year survival rate of up to $40 \%$ and a disease-free period up to 10 years. ${ }^{16}$ There are no standard systemic therapies for patients with intestinal melanoma. Treatment of unresectable metastatic disease can include chemotherapy with dacarbazine, immunotherapy using interleukin-2, interferon alfa, Ipilimumab or biochemotherapy using the combination of both chemotherapy and immunotherapy. In view of extensive metastasis to various lymph nodes our patient had been for planned palliative chemotherapy and follow up.

\section{CONCLUSION}

Primary melanoma of small bowel is rare and usually are metastatic lesions from unidentified or regressed cutaneous primary lesion. Often, it is diagnosed late owing to the vague symptom complex which occurs years after regression of the primary lesion. Thus, a high index of suspicion is necessary, along with a combination of CECT and PET for early diagnosis, appropriate staging and management. Surgical resection is the mainstay of treatment though prognosis after resection of GI melanoma is far worse than cutaneous melanoma.

Funding: No funding sources Conflict of interest: None declared

Ethical approval: Not required

\section{REFERENCES}

1. Chang AE, Karnell LH, Menck HR. The National Cancer Data Base report on cutaneous and noncutaneous melanoma: a summary of 84,836 cases from the past decade. Cancer: Interdiscipl Int J Am Canc Soc. 1998;83(8):1664-78.

2. Sachs DL, Lowe L, Chang AE, Carson E, Johnson TM. Do primary small intestinal melanomas exist? Report of a case. J Am Acad Dermatol. 1999;41(6):1042-4.

3. Lens M, Bataille V, Krivokapic Z. Melanoma of the small intestine. Lancet Oncol. 2009;10(5):516-21.

4. Blecker D, Abraham S, Furth EE, Kochman ML. Melanoma in the gastrointestinal tract. Am J Gastroenterol. 1999;94(12):3427-33.

5. Cheung MC, Perez EA, Molina MA, Jin X, Gutierrez JC, Franceschi D, et al. Defining the role of surgery for primary gastrointestinal tract melanoma. J Gastrointest Surg. 2008;12(4):731-8.

6. Gill SS, Heuman DM, Mihas AA. Small intestinal neoplasms. J Clin Gastroenterol. 2001;33(4):267-82.

7. Mishima Y. Melanocytic and nevocytic malignant melanomas. Cellular and subcellular differentiation. Cancer. 1967;20(5):632-49.

8. Amar A, Jougon J, Edouard A, Laban P, Marry JP, Hillion G. Primary malignant melanoma of the small intestine. Gastroente-rol Clin Biol. 1992;16(4):365.

9. Letsch A, Keilholz U, Schadendorf D, Assfalg G, Asemissen AM, Thiel E, et al. Functional CCR9 expression is associated with small intestinal metastasis. J Invest Dermatol. 2004;122(3):685-90.

10. Bender GN, Maglinte DD, McLarney JH, Rex D, Kelvin FM. Malignant melanoma: patterns of metastasis to the small bowel, reliability of imaging studies, and clinical relevance. Am J Gastroenterol. 2001;96(8):2392-400.

11. Wade TP, Goodwin MN, Countryman DM, Johnson FE. Small bowel melanoma: extended survival with surgical management. Eur J Surg Oncol. 1995;21(1):90-1.

12. Caputy GG, Donohue JH, Goellner JR, Weaver AL. Metastatic melanoma of the gastrointestinal tract: results of surgical management. Arch Surg. 1991;126(11):1353-8.

13. Liang KV, Sanderson SO, Nowakowski GS, Arora AS. Metastatic malignant melanoma of the gastrointestinal tract. In Mayo Clinic Proceedings 2006;81(4):511-16.

14. Tarantino L, Nocera V, Perrotta M, Balsamo G, Schiano A, Orabona P, et al. Primary Small-Bowel Melanoma. J Ultrasound Med. 2007;26:121-7.

15. Tatlidil R, Mandelkern M. FDG-PET in the detection of gastrointestinal metastases in melanoma. Melanoma Res. 2001;11(3):297-301.

16. Schuchter LM, Green R, Fraker D. Primary and metastatic diseases in malignant melanoma of the gastrointestinal tract. Curr Opin Oncol. 2000;12(2):181-5.

Cite this article as: Rajendharan KA, Balakrishnan G, Sundaramurthi S, Krishnaraj B, Sistla SC. Ileal intussusception due to primary intestinal melanoma with generalized lymph node metastases- a rare cause of small bowel obstruction. Int Surg J 2021;8:739-42. 